\title{
Cardiovascular Risk Education and Social Support (CaRESS): Report of a Randomized Controlled Trial from the Kentucky Ambulatory Network (KAN)
}

\author{
Kevin A. Pearce, MD, MPH, Margaret M. Love, PhD, Brent J. Shelton, PhD, \\ Nancy E. Schoenberg, PhD, Mary A. Williamson, RN, BSN, Mary A. Barron, RN, BSN, \\ and Jessica M. Houliban, RD, $M P H$
}

Purpose: Test a practice-based intervention to foster involvement of a relative or friend for the reduction of cardiovascular risk in patients with type 2 diabetes.

Methods: We enrolled in a randomized controlled trial 199 patients and 108 support persons (SPs) from 18 practices within a practice-based research network. All patient participants had type 2 diabetes with suboptimal blood pressure control and were prepared to designate a SP. A subset of the patients also had dyslipidemia. All study visits were conducted at the practice sites where staff took standardized blood pressure measurements and collected blood samples. All patients completed one education session and received newsletters aimed at improving key health behaviors. Intervention group patients included their chosen SP in the education session and the SPs received newsletters.

Results: After 9 to 12 months, the intervention had no significant effect on systolic blood pressure, HbA1C, health-related quality of life, patient satisfaction, medication adherence, or perceived health competence. Power was insufficient to detect an effect on low-density lipoprotein cholesterol. Baseline cardiovascular risk values were not very high, with mean systolic blood pressure at $140 \mathrm{~mm} \mathrm{Hg}$; mean HbA1C at 7.6\%; and mean low-density lipoprotein at $137 \mathrm{mg} / \mathrm{dL}$. Patient health care satisfaction was high.

Conclusion: This practice-based intervention to foster social support for chronic care management among diabetics had no significant impact on the targeted outcomes. (J Am Board Fam Med 2008;21: 269-281.)

Major cardiovascular risk factors among Americans are highly prevalent but poorly controlled, especially among diabetics. Only one fourth of hypertensive Americans have their hypertension under control. ${ }^{1,2}$ The prevalence of dyslipidemia warranting treatment is approximately $29 \%$, and control rates are probably no better than for hypertension. ${ }^{3}$ Approximately 20 million Americans have type 2

This article was externally peer reviewed.

Submitted 10 January 2008; revised 11 April 2008; accepted 14 April 2008.

From the Department of Family and Community Medicine (KAP, MML, MAW, MAB, JMH), the Department of Internal Medicine (BJS), and the Department of Behavioral Science (NES), University of Kentucky College of Medicine, Lexington.

Funding: This project was supported by a grant from the Agency for Healthcare Research and Quality, grant award no. 5R18HS010887-03.

Conflict of interest: none declared.

Corresponding author: Kevin A. Pearce, MD, MPH, Department of Family and Community Medicine, University of Kentucky College of Medicine, Room K-302, Kentucky Clinic, Lexington, KY 40536-0284 (E-mail: kpearce@email. uky.edu). diabetes. ${ }^{4}$ Diabetes triples the risk of symptomatic cardiovascular disease (CVD), which causes two thirds of all deaths among diabetics. ${ }^{5,6}$ Morbidity and mortality are reduced when diabetics use lipidlowering or antihypertensive therapy. ${ }^{2,5,6-8}$ However, the target levels for blood pressure (BP) and low-density lipoprotein (LDL) set for diabetics are especially difficult to achieve. ${ }^{2,7,8}$ Poor adherence to treatment and ineffective patient education often contribute to poor control of cardiovascular risk. $^{9-12}$ Adherence to treatments is especially problematic for diabetics because of their complex treatment regimens. ${ }^{12-15}$

Patients with diabetes typically obtain most of their medical care from primary care providers (PCPs). ${ }^{16}$ Practical strategies for use by PCPs to improve their patients' health behaviors are needed to realize the potential health impacts of powerful treatments. Patient education must be reinforced with other approaches for behavior change to have significant impacts on health. ${ }^{17-19}$ Social support is a key factor in the successful management of 
chronic diseases; it is a strong predictor for treatment adherence and favorable outcomes. ${ }^{9,15,20-22}$ However, most reported interventions used to enhance social support for chronic disease management are too costly and intensive to be feasible for most primary care practices under current reimbursement models. We therefore sought to evaluate the potential of a more practical primary care intervention to enhance social support for chronic disease management.

Potential pathways for social support to influence health outcomes are complex. Because health beliefs have been associated with health behaviors affecting cardiovascular risk, ${ }^{23-26}$ we used the Health Belief Model as a guiding conceptual framework and incorporated theories of self-efficacy. ${ }^{27-31}$ Self-efficacy (perceived health competence), the belief that one has influence over success and that one can succeed, has been positively associated with the likelihood of behavior change. ${ }^{29}$ According to our model, cardiovascular risk factors such as hypertension and dyslipidemia are influenced by health behaviors such as adherence to prescribed diet and exercise, which in turn are influenced by health beliefs such as self-efficacy, perceived susceptibility, disease severity, and barriers. Personal relationships influence pertinent health beliefs. A support person (SP) can serve as a cue to action, reduce barriers to adherence, and promote self-efficacy; or, conversely, the SP might have detrimental effects on health behaviors through negative influences such as nagging. ${ }^{32,33}$ Moreover, the SP's influence may be moderated by factors such as the patient's age, gender, and education, the complexity of prescribed treatments, the patient's overall health status, and insurance coverage. ${ }^{9,34}$

The purpose of this study was to test the effectiveness of an intervention to foster the involvement of a relative or friend as an SP in the control of cardiovascular risk factors in patients with type 2 diabetes. Our intervention was designed for broad and sustainable use in busy primary care practices. We avoided restricting the intervention to patients who had been formally assessed as most likely to benefit from such an intervention because we did not presume that such assessments would routinely occur in practice.

\section{Methods}

Details of our methodology have been published. ${ }^{31}$ This protocol was approved and monitored by the
Medical Institutional Review Board of the University of Kentucky.

\section{Design Overview}

We performed a clustered randomized controlled trial involving 18 primary care practices in the Kentucky Ambulatory Network, a practice-based research network (PBRN). The intervention was designed to educate, motivate, and facilitate patients and their SPs to work together to improve the patients' cardiovascular risk, health-related quality of life (HRQL), and satisfaction with health care. The roles of plausible mediating and moderating factors were evaluated, including medication adherence, basic relationship of patient to SP, quality and degree of SP involvement, patient demographics, history of CVD, health-related self-efficacy, and levels of social support. Participants had 9 to 12 months of follow-up. The study was restricted to patients willing and able to formally involve a SP in the control of the target conditions.

\section{Settings/Locations}

Each patient participant received care at one of 18 PBRN practices, where all study visits occurred. Participating PBRN practices were chosen based on their willingness to collaborate and their distance from the coordinating center. All data were collected at the primary care practices or via telephone. The study was coordinated at the University of Kentucky by Kentucky Ambulatory Network staff.

\section{Patient Eligibility Criteria}

1. Either type 2 diabetes based on chart review according to diagnostic criteria of the American Diabetes Association ${ }^{35}$ or the diagnosis of type 2 diabetes recorded by the PCP along with a HbA1C level $\geq 8.0 \%$, a random serum glucose level $>200 \mathrm{mg} / \mathrm{dL}$, or a current prescription for an antidiabetic drug.

2. Hypertension with suboptimal control, with or without uncontrolled dyslipidemia (see below).

3. Prepared to designate an SP with whom the patient would be in contact for the next 12 months.

4. At least 21 years old and able to give informed consent.

5. Not pregnant or planning to become pregnant within the next 12 months. 
6. Planning to be available for follow-up for at least the next 12 months.

We defined hypertension with suboptimal control in diabetics as a mean systolic BP (SBP) $>129$ $\mathrm{mm} \mathrm{Hg}$ based on the last 2 visits recorded in the chart, or on the highest 2 readings in the last 6 months, and a mean SBP $>129 \mathrm{~mm} \mathrm{Hg}$ based on 3 standardized readings taken at the screening study visit. (SBP was used as the sole BP criterion to facilitate hypothesis-testing and sample-size calculations.)

We defined dyslipidemia with suboptimal control in diabetics as LDL $>100 \mathrm{mg} / \mathrm{dL}$ and confirmation of suboptimal control based on fasting lipid profile done at baseline. (LDL was used as the sole lipid criterion to facilitate hypothesis-testing and estimation of required sample size.)

These definitions for suboptimal control of hypertension and dyslipidemia match the recommended thresholds for starting or altering drug therapy in diabetics. $2,5,36$

\section{Support Person (SP) Eligibility Criteria}

1. Adult able to give informed consent.

2. Regular (at least weekly) contact with the patient expected throughout the coming 12 months.

3. Chosen by the patient based on advice for effective SP provided by the investigators (can arrange at least weekly contact, SP willing to come to study visits, likely to be supportive of patient in improving health behaviors).

\section{Identification and Enrollment of Participants}

Potential participants were identified using each practice's billing data, followed by medical record review. To minimize selection bias, patients were not recruited during visits to the PCP. Each practice provided a list of all adult patients who had made at least one visit in the past 2 years and who had ever had diabetes coded as a diagnosis. Study personnel then reviewed all of these patient charts for eligibility. Potentially eligible patients were sent an explanatory letter about the study and signed by the PCP. During follow-up recruiting by telephone, study personnel worked down a randomly ordered list of these letter recipients until the a priori target number of 25 patients from each practice had been scheduled for a screening visit or until repeated attempts had been made to reach all patients on the list. Each patient participant was re- quired to name a potential SP and all were instructed on criteria for choosing an SP. Study personnel enrolled the designated SP for each patient participant randomized to the intervention group. The SPs designated by patients randomized to the control group were not contacted by study personnel.

\section{Randomization Methods and Study Groups}

To avoid contamination, randomization was done at the practice level, and all participants at a given practice were assigned to the same treatment group. The project statistician maintained sealed envelopes containing group assignment. Masking and blocked randomization were used to prevent recruitment bias and unbalanced allocation. All patient participants at each practice were enrolled before group assignment was unmasked. Once group assignment was revealed, each patient was informed by phone; if assigned to involve a SP, the patient was instructed to bring the SP to the next study visit, which was the actual intervention visit.

Subjects were randomized to the control group or one of 2 intervention groups (A and B). Our design had 2 intervention groups to facilitate exploration of mechanisms and predictors of the intervention's impacts while minimizing the Hawthorne Effect. Participants in intervention group B had more contact with study personnel, who collected data to more deeply probe the mechanisms through which involvement of an SP might affect outcomes, but they did not receive any additional education or coaching. We planned to test our quantitative outcomes-related hypotheses through comparisons of the control group with intervention group A.

\section{Sample Size}

Our a priori sample size calculations were based on the plan to test our main hypotheses by comparing the control group with intervention group A. We used published variance data on SBP, LDL cholesterol, and HRQL scores to support sample size calculations.

\section{Hypotheses Used to Drive Sample Size Calculations}

After the intervention and controlling for baseline values, the following were hypotheses used to drive sample size calculations: 
- Mean SBP will be at least $8 \mathrm{~mm} \mathrm{Hg}$ lower in the intervention group than in the control group.

- Mean LDL cholesterol will be at least $15 \mathrm{mg} / \mathrm{dL}$ lower in the intervention group than in the control group.

- HRQL, as measured by the Medical Outcomes Study Short Form (SF-36) physical composite score, will be at least 10 points better (on a 100-point scale) in the intervention group than in the control group.

- We set our targeted sample size to detect at least these differences between groups with at least $90 \%$ power and an $\alpha$ level of 0.05 . These calculations led to a targeted sample size of $100 \mathrm{pa}-$ tients per group completing the study.

\section{Timelines}

The entire study lasted 4 years (2002-2006). Participants at the first 15 practices were followed for 12 months; those from the last 3 practice sites had 9 months of follow-up.

\section{Intervention}

The intervention was designed to foster the formal involvement of a friend or relative as an SP to help the patient lower cardiovascular risk. It consisted of one patient/SP education session followed by 4 quarterly "newsletters." Patients in the intervention groups had their SP join them for a 30-minute individualized patient education session with a Registered Nurse patient educator, delivered at the practice site. The session focused on cardiovascular risk reduction advice for the patient and ways that the SP could help. Guidelines for effective patient SP interactions were included, stressing the SP role as facilitator and advising that the patient should be responsible for his/her own health behaviors. Specific strategies suggested to the SP included accompanying the patient during doctor visits, reviewing medication instructions and supplies with the patient, exercising with the patient, grocery shopping with the patient, and talking with the patient about his or her concerns and specific barriers to cardiovascular risk factor control. After the education session, 4 quarterly patient education newsletters about cardiovascular risk factor control were mailed to the patients; similar newsletters focusing on facilitative strategies were mailed to the SPs.

Patients randomized to the control group received an individual 30-minute patient education session with a Registered Nurse patient educator and received the same 4 patient newsletters as sent to intervention group patients, but control group patients did not have formal involvement of their $\mathrm{SP}$ in the program.

All education sessions followed a standardized curriculum guided by a notebook given to the participants. Notebook materials came from the American Heart Association, the National Institutes of Health, the American Diabetes Association, and the American Academy of Family Physicians, and were selected for accuracy, clarity, brevity, reading level at or below 8 th grade, appropriate focus, and avoidance of confusing or conflicting recommendations. Information about CVD among diabetics, hypertension, high cholesterol, diet and exercise guidelines with examples, the importance of medication adherence and tips for improving it, advice on communicating with health care providers and keeping appointments, and advice on making lifestyle changes related to tobacco avoidance, diet, and exercise was included in the notebook. Intervention group materials included advice on how to help someone else make lasting improvements in their health behaviors. The newsletters reinforced the same curriculum, with each newsletter focusing on one or 2 of these topics.

Participants' PCPs were aware of which patients were enrolled because they received study-related laboratory test reports (the same tests, regardless of study group assignment), but our patient-oriented intervention did not include any instructions or facilitation for the PCPs related to evaluation or management of cardiovascular risk.

\section{Study Visit Protocol}

All visits were performed at the practice sites. Each patient participant made 7 study visits over a 12 month period: 3 visits at baseline, 2 visits at 6 months after randomization, and 2 visits at 12 months after randomization. In intervention group A, SPs accompanied their patients to one visit at baseline and one at 12 months. SPs in intervention group B made one visit at baseline, one at 6 months, and one at 12 months. Patient and SP education activities occurred during visit 3. All other visits were strictly for data collection. Paired visits allowed repeated BP and LDL measurements to dampen intraperson short-term variability. 
Table 1. Data Collected for Each Participant, by Study Visit

\begin{tabular}{|c|c|c|c|c|c|c|c|}
\hline & Visit 1 & Visit 2 & Visit 3 & Visit 4 & Visit 5 & Visit 6 & Visit 7 \\
\hline Time since randomization* (months) & Baseline & Baseline & Baseline & 6 & 6 & 12 & 12 \\
\hline Resting BP & $\mathrm{A}, \mathrm{B}, \mathrm{C}$ & $\mathrm{A}, \mathrm{B}, \mathrm{C}$ & $\mathrm{A}, \mathrm{B}, \mathrm{C}$ & $\mathrm{A}, \mathrm{B}, \mathrm{C}$ & $\mathrm{A}, \mathrm{B}, \mathrm{C}$ & $\mathrm{A}, \mathrm{B}, \mathrm{C}$ & $\mathrm{A}, \mathrm{B}, \mathrm{C}$ \\
\hline Fasting lipid profile & & $\mathrm{A}, \mathrm{B}, \mathrm{C}$ & $\mathrm{A}, \mathrm{B}, \mathrm{C}$ & $\mathrm{A}, \mathrm{B}, \mathrm{C}$ & $\mathrm{A}, \mathrm{B}, \mathrm{C}$ & $\mathrm{A}, \mathrm{B}, \mathrm{C}$ & $\mathrm{A}, \mathrm{B}, \mathrm{C}$ \\
\hline $\mathrm{HbA1C}$ & & $\mathrm{A}, \mathrm{B}, \mathrm{C}$ & & $\mathrm{A}, \mathrm{B}, \mathrm{C}$ & & $\mathrm{A}, \mathrm{B}, \mathrm{C}$ & \\
\hline CVD history & $\mathrm{A}, \mathrm{B}, \mathrm{C}$ & & & & & & \\
\hline Interval CVD history & & & & & $\mathrm{A}, \mathrm{B}, \mathrm{C}$ & & $\mathrm{A}, \mathrm{B}, \mathrm{C}$ \\
\hline Medication review & $\mathrm{A}, \mathrm{B}, \mathrm{C}$ & & & & $\mathrm{A}, \mathrm{B}, \mathrm{C}$ & & $\mathrm{A}, \mathrm{B}, \mathrm{C}$ \\
\hline Medication adherence & & & $\mathrm{A}, \mathrm{B}, \mathrm{C}$ & & B & & $\mathrm{A}, \mathrm{B}, \mathrm{C}$ \\
\hline HRQL & $\mathrm{A}, \mathrm{B}, \mathrm{C}$ & & & & $\mathrm{A}, \mathrm{B}, \mathrm{C}$ & & $\mathrm{A}, \mathrm{B}, \mathrm{C}$ \\
\hline Healthcare satisfaction & $\mathrm{A}, \mathrm{B}, \mathrm{C}$ & & & & & & $\mathrm{A}, \mathrm{B}, \mathrm{C}$ \\
\hline Health-related self-efficacy & $\mathrm{A}, \mathrm{B}, \mathrm{C}$ & & & & B & & B \\
\hline Social network & $\mathrm{A}, \mathrm{B}, \mathrm{C}$ & & & & $\mathrm{B}$ & & $\mathrm{A}, \mathrm{B}, \mathrm{C}$ \\
\hline SP qualities/involvement & $\mathrm{A}, \mathrm{B}, \mathrm{C}$ & & & & B & & $\mathrm{A}, \mathrm{B}, \mathrm{C}$ \\
\hline Demographics & $\mathrm{A}, \mathrm{B}, \mathrm{C}$ & & & & $\mathrm{A}, \mathrm{B}, \mathrm{C}$ & & $\mathrm{A}, \mathrm{B}, \mathrm{C}$ \\
\hline
\end{tabular}

Patient education session occurred during visit 3.

*Randomization occurred after visit 2.

$\mathrm{BP}$, blood pressure; CVD, cardiovascular disease; HRQL, health-related quality of life; SP, support person; A, intervention group A; $\mathrm{B}$, intervention group $\mathrm{B}$; $\mathrm{C}$, control group.

\section{Data Collection Protocol}

The data collection protocol is shown in Table 1. Standardized BP readings and phlebotomy were performed by nurses and medical assistants employed at each practice. All blood samples were analyzed at one university laboratory. All other data were collected by study coordinators who went to each practice site. All nurses and medical assistants who took BP measurements completed training and certification in standardized BP measurement, following American Heart Association guidelines. Training and certification were repeated after 6 months. BP readings were done in triplicate to calculate average $\mathrm{BP}$ for that visit. LDL cholesterol levels were collected as paired samples on different days to derive average levels for baseline and follow-up periods and were limited to the subset of patients whose most recent LDL on record was elevated. Participants with no LDL level on record did not have an LDL level drawn for this study. Instruments used to collect all other data are shown in Table 2.

\section{Analytic Methods}

Intervention group differences in baseline characteristics were assessed using mixed effects models where the clinic was treated as a random effect with the patient nested within the clinic, adjusting for

Table 2. Method and/or Instrument(s) Used for Non-physiologic Measures

\begin{tabular}{ll}
\hline Measure & \multicolumn{1}{c}{ Method(s)/Instrument(s) } \\
\hline CVD history & Patient structured interview with research staff \\
Interval CVD history & Patient structured interview with research staff \\
Medication review & Patient structured interview with research staff \\
Medication adherence & Medication Adherence Questionnaire \\
HRQL & SF-36 Health Survey \\
Healthcare satisfaction & Patient Healthcare Satisfaction Survey \\
Self-efficacy & Perceived telephone \\
Social support network & Self-report at baseline on whether person(s) already helping patient with CVD \\
& risk management and person patient would ask to be SP (friend vs. relative) \\
SP qualities/involvement & Social Support for Intervention Survey \\
Demographics & Patient structured interview with research staff \\
\hline
\end{tabular}

CVD, cardiovascular disease; HRQL, health-related quality of life; SP, support person. 
the intraclass correlation coefficient (ICC) within the clinic. $^{42}$ The ICCs for study outcomes calculated across the 18 clinic sites ranged from 0.0108 (satisfaction with health care) to 0.1046 (SBP).

Baseline characteristics and outcomes did not differ between the 2 intervention groups (A and B). The only differences between these 2 groups were in assessment, not intervention (see Table 1). Extra surveys were administered in group B to probe the mechanisms through which involvement of an SP might affect outcomes, but these participants did not receive any extra education, encouragement, or coaching compared with group A. To improve study power, we therefore collapsed groups A and $\mathrm{B}$ into a single intervention group for comparisons to the control group.

Main models to assess for differences between the intervention and control groups were fit to each individual outcome, adjusting for baseline values and ICCs. A second set of outcome models included adjustments for age, race, sex, employment, education level, health insurance, CVD event composite score, medication adherence level, perceived health competence, alcohol use, current smoking status, level of health-related social support, relationship with SP, and self-reported histories of high BP and high cholesterol.

The Statistical Analysis System procedure PROC MIXED (SAS Institute, Inc., Cary NC) was used to assess intervention effects on the outcome data while accounting for missing outcome measures because of drop-outs or sporadically missing data. Post hoc power calculations were conducted (see Results, below).

\section{Results}

\section{Participant Flow and Baseline Characteristics}

We enrolled 199 diabetic patients and 108 SPs from among 18 primary care practices and followed them for 9 to 12 months after randomization. Medical records on 2608 diabetic adults were screened; 1318 met initial eligibility criteria, 336 patients completed a screening visit, 233 patients were consented and enrolled, and 199 patients plus 108 consenting SPs completed the education session. The 34 patients "lost" before completing their education session included 4 who decided after consenting that they could not bring an SP and 30 who dropped out before completing the education session.
Baseline characteristics of the 199 patients are shown in Table 3. Randomization resulted in even distribution of values across the study groups; however, health insurance status differed: the control group had more privately insured patients (70\%) than intervention group A (53\%) or group B (52\%), with group A having the most uninsured patients (14\%). In addition, more patients reported having high cholesterol in the control group (80\%) than in the intervention groups A or B $(67 \%, 55 \%)$. SP characteristics are shown in Table 4.

Given the absence of significant baseline differences between intervention groups A and B, we pooled these into a single intervention group for our analyses; this allowed us to achieve sufficient power levels for all main outcomes except LDL cholesterol level.

\section{Main Outcomes}

Table 5 shows the main outcomes at 6 months and 9 to 12 months after randomization. For each outcome compared across groups, $2 P$ values are given. The first is adjusted only for baseline value and the ICC. The second $P$ value is also adjusted for potential mediating or moderating factors (see Table 5 footnotes). Diminishing sample sizes over time reflect the drop-out rates.

\section{Effects of Intervention}

SBP fell in control and intervention groups, probably partly because of regression to the mean. The patient education received by all groups may have also played a role. There were no significant between-group differences in the change in SBP from baseline to 6 months and 9 to 12 months after randomization. There was also no significant effect of group assignment on diastolic BP (not shown).

LDL fell in the control and intervention groups, also probably partly because of regression to the mean. The patient education received by all groups may have also played a role. There were no statistically significant between-group differences in the change in LDL.

There were no significant between-group differences in the change in $\mathrm{HbAlc}$ from baseline to 9 to 12 months after randomization. There was a trend toward a slightly greater reduction in $\mathrm{HbA1C}$ levels in the control group at 6 months.

There were no significant between-group differences in the change in the Physical Composite Scale or the Mental Composite Scale of the SF-36 


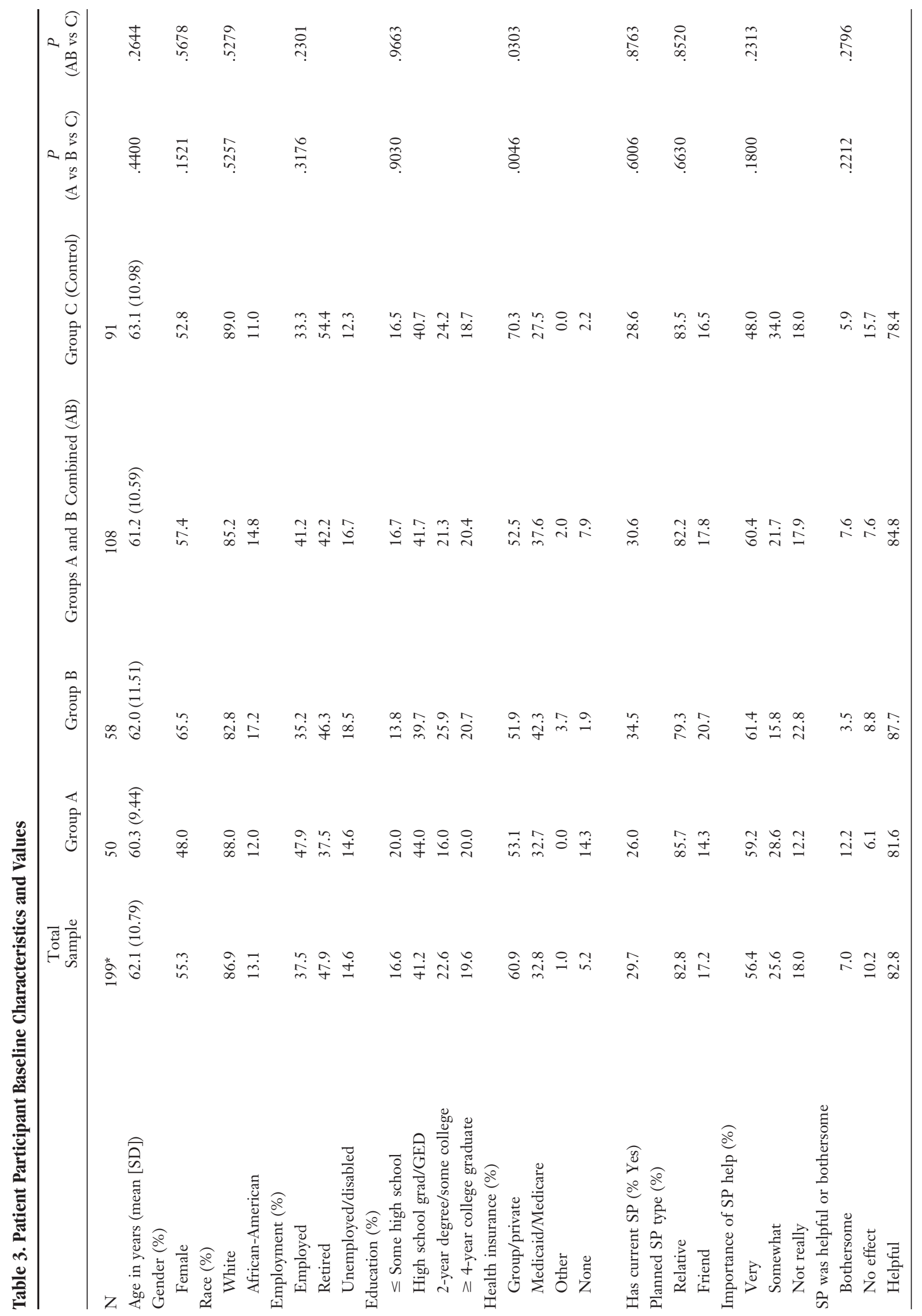




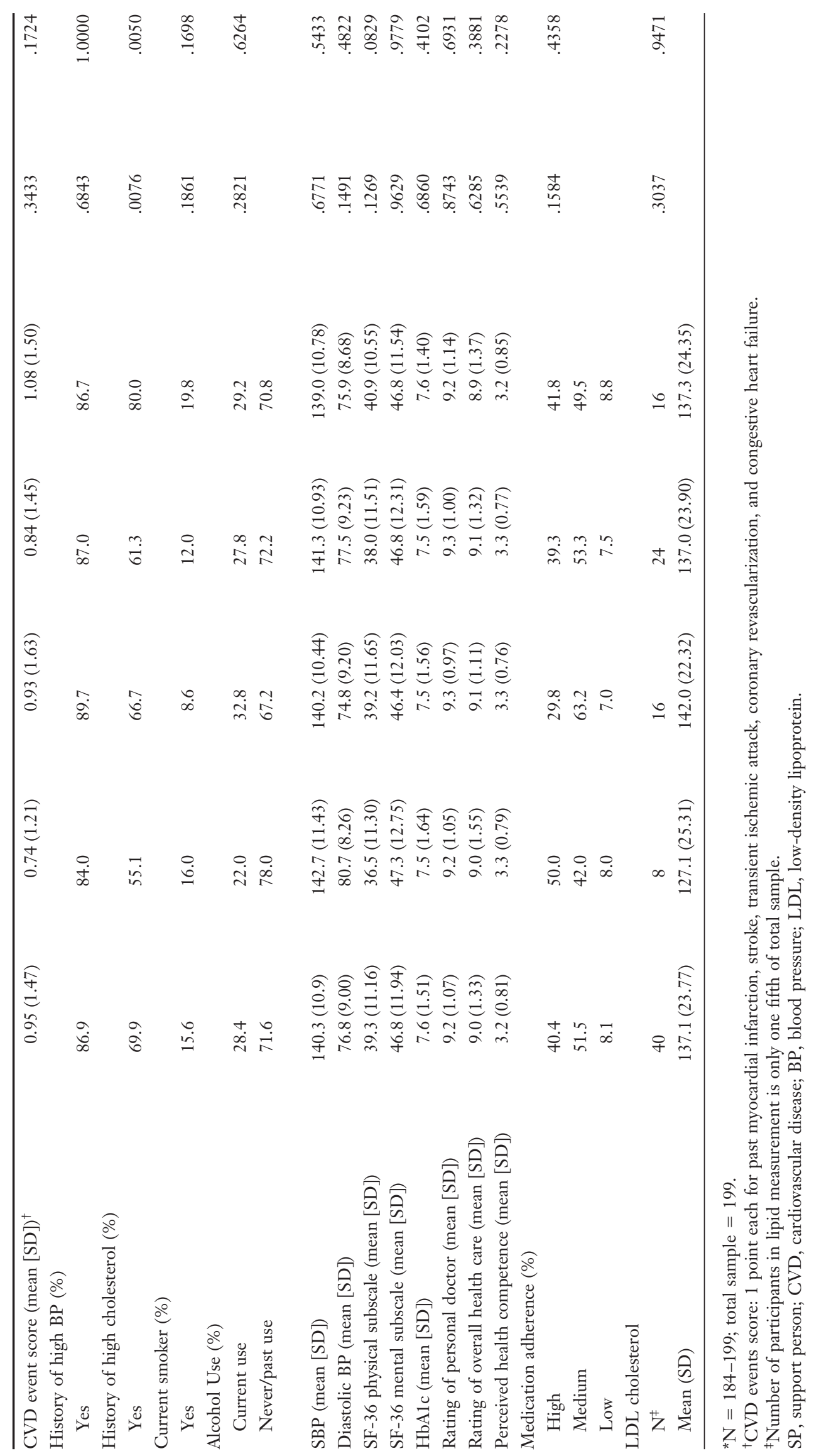


Table 4. Support Person Characteristics

\begin{tabular}{lc}
\hline Age in Years (mean [SD]) & $55(14.5)$ \\
Female (\%) & 75 \\
Race (\%) & 86 \\
White & 13 \\
African-American & 1 \\
Asian & \\
Occupation status (\%) & 48 \\
Employed & 34 \\
Retired & 18 \\
Unemployed & \\
Education Level (\%) & 17 \\
$\quad<$ High school & 35 \\
High school grad or GED & 25 \\
Some college & 23 \\
$\geq 4-$ year degree & \\
Relationship to patient (\%) & 51 \\
Spouse & 16 \\
Child & 5 \\
Mother & 11 \\
Other relative & 17 \\
Friend and/or neighbor & \\
\hline
\end{tabular}

from baseline to 6 months or 9 to 12 months after randomization.

Satisfaction with the PCP improved slightly in both groups, whereas satisfaction with health care overall declined slightly in both groups. Betweengroup differences became statistically nonsignificant after the described adjustments, and were not clinically significant on a scale of 1 to 10 .

\section{Power Considerations for Main Outcomes}

Recruitment lagged behind our design target of 300 completed patient studies, leaving this study with marginal power to avoid type II errors in some of our outcomes analyses, and no power to detect differences in LDL levels. Ultimately, we had $80 \%$ power $(\alpha=0.05)$ to detect differences between the control group and the combined intervention groups (A and B) (see Table 6).

\section{Potential Cognitive Bebavioral Mechanisms Linking the Intervention and the Main Outcomes}

Two mechanisms were analyzed as potential moderators of the intervention effects on the main outcomes and as proximal outcomes themselves. Baseline levels of neither self-efficacy nor medication adherence were found to moderate intervention effects for any of the main outcomes. Furthermore, there were no significant between-group differ- ences in the change in self-efficacy or medication adherence from baseline to 6 or 12 months after randomization.

\section{Discussion}

This randomized, controlled trial of a practicebased intervention intended to foster the formal involvement of a friend or relative in the care of adults with type 2 diabetes showed no significant effects on the main outcomes of interest, including SBP, LDL, HbA1C, HRQL, and patient satisfaction. The intervention also had no significant effect on perceived self-efficacy in managing one's own health or on medication adherence.

There are a few plausible explanations for this lack of impact. The first is that the intervention was not robust enough to bring about positive health behavior change beyond the effects of patient education. A single educational session with a nurse, followed by reinforcing newsletters, seems to be insufficient to foster social support at a level that leads to improved health behaviors. Second is the possibility of ceiling or floor effects within our sample. Although SBP $>129 \mathrm{~mm} \mathrm{Hg}$ and LDL $>100 \mathrm{mg} / \mathrm{dL}$ determined eligibility for study entry, the mean levels of these risk factors in participants at baseline were not very high $(140 \mathrm{~mm} \mathrm{Hg}$ and 137 $\mathrm{mg} / \mathrm{dL}$, respectively). Having type 2 diabetes was an entry criterion, but $\mathrm{HbA} 1 \mathrm{C}$ level was not, and the mean baseline HbA1C was only $7.6 \%$. Thus, on the whole, these patients and their physicians may have felt less motivation for improving these cardiovascular risk factors than would be the case for patients with worse control. Furthermore, these patients' satisfaction with their PCPs and their overall health care was very high at baseline, leaving almost no room for improvement. Third, because this intervention required participants to name a potential SP, their overall social support may have been stronger than would be seen in the general population of adult diabetics; thus there may have been less room for improvement in support for cardiovascular risk reduction. We have no data to support or refute this possibility.

\section{Limitations}

Lagging enrollment led to a smaller sample size than was planned and required us to shorten the follow-up period to 9 months for the last $42 \mathrm{pa}$ tients. This resulted in insufficient power to mea- 
Table 5. Primary Outcomes: Adjusted* and Unadjusted ${ }^{\dagger}$ Group Differences at 6 Months and 9 to 12 Months After Baseline

\begin{tabular}{|c|c|c|c|c|c|}
\hline & $\begin{array}{l}\text { Baseline } \\
(\text { mean }[\mathrm{n}])\end{array}$ & $\begin{array}{l}6 \text { Months } \\
\text { (mean }[\mathrm{n}] \text { ) }\end{array}$ & $\begin{array}{l}9 \text { to } 12 \text { Months } \\
\text { (mean }[\mathrm{n}])\end{array}$ & $\begin{array}{c}\text { Change from Baseline } \\
\text { to } 6 \text { Months }\end{array}$ & $\begin{array}{l}\text { Change from Baseline } \\
\text { to } 9 \text { to } 12 \text { Months }\end{array}$ \\
\hline \multicolumn{6}{|l|}{ Systolic BP (mmHg) } \\
\hline Intervention group $\mathrm{AB}$ & $141.3(108)$ & $135.5(92)$ & $134.0(81)$ & -5.8 & -7.3 \\
\hline Control group C & $139.0(91)$ & $133.6(74)$ & $133.8(60)$ & -6.4 & -5.2 \\
\hline Unadjusted $P$ for $\mathrm{AB}$ vs $\mathrm{C}$ & .5433 & .3836 & .9427 & & \\
\hline Adjusted $P$ for $\mathrm{AB}$ vs $\mathrm{C}$ & na & .4969 & .6475 & & \\
\hline \multicolumn{6}{|l|}{ HbA1C (\%) } \\
\hline Intervention group $\mathrm{AB}$ & $7.5(106)$ & $8.3(87)$ & $7.4(74)$ & 0.8 & -0.1 \\
\hline Control group C & $7.6(85)$ & $7.8(63)$ & $7.4(63)$ & 0.2 & -0.2 \\
\hline Unadjusted $P$ for $\mathrm{AB}$ vs $\mathrm{C}$ & .4102 & .0567 & .6440 & & \\
\hline Adjusted $P$ for $\mathrm{AB}$ vs $\mathrm{C}$ & na & .0429 & .9164 & & \\
\hline \multicolumn{6}{|l|}{ SF-36 Physical composite score } \\
\hline Intervention group $\mathrm{AB}$ & $38.0(107)$ & $42.7(84)$ & $41.4(74)$ & 4.7 & 3.4 \\
\hline Control group C & $40.9(88)$ & $42.6(74)$ & $41.6(72)$ & 1.7 & 0.7 \\
\hline Unadjusted $P$ for $\mathrm{AB}$ vs $\mathrm{C}$ & .0829 & .4145 & .4345 & & \\
\hline Adjusted $P$ for $\mathrm{AB}$ vs $\mathrm{C}$ & na & .9598 & .9056 & & \\
\hline \multicolumn{6}{|l|}{ SF-36 mental composite score } \\
\hline Intervention group $\mathrm{AB}$ & $46.8(107)$ & $42.7(84)$ & $45.7(74)$ & -4.1 & -1.1 \\
\hline Control group C & $46.8(88)$ & $40.1(74)$ & $47.9(72)$ & -6.7 & 1.1 \\
\hline Unadjusted $P$ for $\mathrm{AB}$ vs $\mathrm{C}$ & .9779 & .2666 & .5200 & & \\
\hline Adjusted $P$ for $\mathrm{AB}$ vs $\mathrm{C}$ & na & .2187 & .2916 & & \\
\hline \multicolumn{6}{|l|}{ Rate of primary doctor ${ }^{\S}$} \\
\hline Intervention group $\mathrm{AB}$ & $9.3(98)$ & & $9.5(71)$ & & 0.2 \\
\hline Control group C & $9.2(86)$ & & $9.3(67)$ & & 0.1 \\
\hline Unadjusted $P$ for $\mathrm{AB}$ vs $\mathrm{C}$ & .6931 & & .0255 & & \\
\hline Adjusted $P$ for $\mathrm{AB}$ vs $\mathrm{C}$ & na & & .6372 & & \\
\hline \multicolumn{6}{|l|}{ Rating of overall health care ${ }^{\S}$} \\
\hline Intervention group $\mathrm{AB}$ & $9.3(98)$ & & $8.3(71)$ & & -1.0 \\
\hline Control group C & $9.2(86)$ & & $8.5(67)$ & & -0.7 \\
\hline Unadjusted $P$ for $\mathrm{AB}$ vs $\mathrm{C}$ & .6931 & & .0255 & & \\
\hline Adjusted $P$ for $\mathrm{AB}$ vs $\mathrm{C}$ & na & & .6709 & & \\
\hline \multicolumn{6}{|l|}{ LDL cholesterol ${ }^{\|}$} \\
\hline Intervention group $\mathrm{AB}$ & $137.0(24)$ & $139.4(18)$ & $135.4(18)$ & 2.4 & -1.6 \\
\hline Control group C & $137.3(16)$ & $130.5(11)$ & $110.6(11)$ & -6.6 & -26.7 \\
\hline $\begin{array}{l}\text { Unadjusted } P \text { for } \mathrm{AB} \text { vs } \mathrm{C} \\
\text { Adjusted } P \text { for } \mathrm{AB} \text { vs } \mathrm{C}\end{array}$ & .9471 & .6716 & .3238 & & \\
\hline
\end{tabular}

*Unadjusted: $P$ adjusted only for clustering of patient within clinic and baseline outcome variable values for 6 months and 12 month comparisons.

${ }^{\dagger}$ Adjusted: $P$ adjusted for clustering, baseline outcome values, age, sex, race, education, employment status, health insurance, whether already had an SP, relationship with SP, baseline values for medication adherence, perceived health competence, patient self-report of history of high BP, history of high cholesterol, smoking status, alcohol use and history of cardiovascular events score (1 point each for previous myocardial infarction, stroke, transient ischemic attack, coronary revascularization, and congestive heart failure). Given the limited sample size for LDL measurements, no adjusted $P$ are presented.

${ }^{\ddagger}$ SF-36 subscale possible range is $0-100$.

${ }^{\$}$ Doctor and healthcare satisfaction scales each have possible range from $0-10$.

"The smaller subsample of patients with measured LDL permitted analyses controlling only for baseline LDL and clustering within clinic, not for adjusted analyses with additional covariates.

BP, blood pressure; LDL, low-density lipoprotein, SP, support person.

sure any significant effect on LDL and insufficient power to detect very small differences in other outcomes. Data were combined for participants who were followed for 9 months and 12 months. Foreshortened follow-up of the last 42 subjects might be expected to falsely skew the results toward 


\begin{tabular}{lcc}
\hline Outcome & Detectable Difference at 6 mo & Detectable Difference at 9 to 12 mo \\
\hline Systolic BP & $10 \mathrm{mmHg}$ & $12 \mathrm{mmHg}$ \\
LDL cholesterol & $59 \mathrm{mg} / \mathrm{dl}$ & $72 \mathrm{mg} / \mathrm{dl}$ \\
HbA1C (\%) & 1.3 percentage points & 1.5 percentage points \\
SF-36 HRQL (100-pt scale) & 6.5 points & 6.9 points \\
Satisfaction with doctor (scale 0-10) & na & 1.3 points \\
Satisfaction with healthcare (scale 0-10) & na & 3.0 points \\
\hline
\end{tabular}

BP, blood pressure; LDL, low-density lipoprotein; HRQL, health-related quality of life.

a lasting intervention effect beyond 6 months, but we saw no effects at 9 to 12 months anyway. Nonrandom selection of participating practices might have led to unreliable results, but because this intervention was patient-oriented, not practiceoriented, it is unlikely that our results would differ if the study were repeated in a random sample of practices.

\section{Conclusions}

The prevention of symptomatic CVD is heavily dependent on health behaviors. Despite ample evidence that social support is positively associated with health and with health behaviors there is little published research about fostering social support to improve chronic disease management in the absence of an acute event, such as after a myocardial infarction. Our lack of impact with an approach that could be used in busy primary care practices is disappointing, but our study is importantly distinguished from those that used more intensive interventions that would be impractical for primary care practices. Other recent practical intervention trials $^{43-45}$ have also failed to link fostering social support with positive health outcomes, such as improved glycemic control or serum cholesterol levels. As in our research, these studies focused on improving social support from family members and friends for the improvement of chronic disease management by using interventions that might be applicable in busy medical practices.

In contrast, studies showing favorable effects used more intense and/or prolonged social support interventions that focused on peer patient group visits, ${ }^{45,46}$ existing strong social support systems, ${ }^{47}$ and other patient education and support group sessions. ${ }^{45,48}$ Interventions combining social support with diet, exercise, and stress management techniques had positive results. ${ }^{45,46,48}$ These interven- tions included various combinations of weekly phone calls, weekly or monthly meetings, retreats, and regular group sessions with dietitians, exercise physiologists, nurses, and stress management specialists.

Practical and powerful methods that busy PCPs can use to foster sustained positive health behavior change in patients who have asymptomatic but dangerous chronic conditions are still needed. Even though the relatively successful approaches summarized above are not feasible for most primary care practices under current payment systems in the United States, PCPs may be able to guide the development of such programs and facilitate the involvement of their patients in them. Promising models that deserve further development and investigation are group visits for chronic care, ${ }^{49,50}$ improved integration of primary care services with work-place wellness programs and/or with chronic disease management programs offered by health insurers, ${ }^{51}$ and providing professional guidance to community programs, such as faith-based health improvement initiatives. ${ }^{52}$

The most powerful solutions may lie in new models for health care reimbursement. Use of care teams coordinated through a primary care medical home and supported by a novel business model has shown promise for improving chronic disease management and deserves further investigation. ${ }^{53}$ Any successful strategy for sustained improvements in such health behaviors may ultimately hinge on empowering, motivating, and equipping people to take greater proactive responsibility for their health.

The authors wish to thank Jenny Carey for her excellent work on data entry for computerized analyses. We also wish to thank the following central Kentucky physicians and practices for their contributions to this research: Berea Primary Care Clinic, Berea White House Clinic, Bluegrass Clinic-Stanford Family Med- 
icine and Obstetrics PSC, Bluegrass Medical Group PSC, Georgetown Family Physicians-Drs. Wechman and Preston, Capital Medical Group, Capital Family Physicians PSC, Central Internal Medicine, Dartt and Hurt PSC, Family Medicine Clinic of Danville, Family Practice Associates of Lexington PSC, Neil Farris MD, Gus Bynum MD PSC, Kentucky Clinic North, Kentucky Clinic South, Scott County Family Physicians, UK Family Medical Center.

\section{References}

1. Fields LE, Burt VL, Cutler JA, Hughes J, Roccella EJ, Sorlie P. The burden of adult hypertension in the United States 1999 to 2000: a rising tide. Hypertension 2004;44:398-404.

2. Chobanian AV, Bakris GL, Black HR, et al. The Seventh Report of the Joint National Committee on Prevention. Detection, Evaluation, and Treatment of High Blood Pressure: the JNC 7 report. JAMA 2003;289:2560-72.

3. Sempos CT, Cleeman JI, Carroll MD, et al. Prevalence of high blood cholesterol among US adults. JAMA 1993;269:3009-14.

4. Laine C, Goldmann DR, Sox HC. In the clinic. Type 2 diabetes. Ann Intern Med 2007;146:70.

5. Grundy SM, Ckleeman JI, Merz CMB, et al. Implications for the National Cholesterol Education Program Adult Treatment Panel III guidelines. Circulation 2004;110:227-39.

6. Curb JD, Pressel SL, Cutler JA, et al. For the systolic Hypertension in the Elderly Program Cooperative Research Group. Effect of diuretic-based antihypertensive treatment on cardiovascular disease risk in older diabetic patients with isolated systolic hypertension. JAMA 1996;276:1886-92.

7. Turner R, Holman R, Stratton I, et al. High blood pressure control and risk of macrovacscular and microvascular complications in type 2 diabetes: UKPDS 38. UK Prospective Diabetes Study Group. BMJ 1998;317:703-13.

8. Pyorala K, Pedersen TR, Kjekshus J, et al. Cholesterol lowering with simvastation improves prognosis of diabetes patient with coronary heart disease. Diabetes Care 1997;20:614-20.

9. Dunbar-Jacob J, Dwyer K, Dunning J. Compliance with antihypertensive regimen: a review of the research in the 1980s. Ann Behav Med 1991;13:31-9.

10. Sanson-Fisher RW, Clover K. Compliance in the treatment of hypertension. Am J Hypertens 1995;8: $82 \mathrm{~S}-88 \mathrm{~S}$.

11. Avorn J, Monette J, Lacour A, et al. Persistence of use of lipid-lowering medications. JAMA 1998;279: $1458-62$.

12. Harris R, Linn MW. Health beliefs, compliance, and control of diabetes mellitus. SMJ 1985;78:162-6.

13. Kern RM, Penick JM, Hamby RD. Prediction of diabetic adherence using the BASIS-A inventory. Diabetes Educ 1996;22:367-73.

14. Glasgow RE, Hampson SE, Strycker LA, Ruggiero
L. Personal-model beliefs and social-environmental barriers related to diabetes self-management. Diabetes Care 1997;20:556-61.

15. Schlenk EA, Hart LK. Relationship between health locus of control, health value, and social support and compliance of person with diabetes mellitus. Diabetes Care 1984;7:556-74.

16. Larson SL, Machlin SR, Nixon A, et al. Healthcare in urban and rural areas, 1998-2000. MEPS Chartbook 13, AHRQ Pub No. 04-0050. Rockville (MD): Agency for Healthcare Quality and Research; 2004.

17. Webb PA. Effectiveness of patient education and psychosocial counseling in promoting compliance and control among hypertensive patients. J Fam Prac 1980;10:1047-55.

18. Shepherd J, Alcalde V, Befort P. International comparison of awareness and attitudes towards coronary risk factor reduction: the HELP study. J Cardiovasc Risk 1997;4:373-84.

19. Bruckert E, Simonetta C, Giral P. Compliance with fluvastatin treatment: characterization of the noncompliant population within a population of 3845 patients with hyperlipidemia. J Clin Epidemiol 1999; 52:5589-94.

20. Garay-Sevila ME, Nava LE, Malacara JM, et al. Adherence to treatment and social support in patients with non-insulin dependent diabetes mellitus. J Diabetes Complications 1995;9:81-6.

21. Morisky DE, DeMuth NM, Field-Fass M, et al. Evaluation of family health education to build social support for long-term control of high blood pressure. Health Educ Quart 1985;12:35-50.

22. Berkman LF. The relationship of social networks and social support to morbidity and mortality. In: Cohen S, Syme SL, editors. Social Support and Health. Orlando, FL: Academic Press, Inc. 1985; 241-61.

23. Jones PK, Jones SL, Katz J. Improving follow-up among hypertensive patients using a health belief model intervention. Arch Intern Med 1987;147:155760.

24. Mirotznik J, Feldman L, Stein R. The health belief model and adherence with a community centerbased, supervised coronary heart disease exercise program. J Community Health 1995;20:233-47.

25. Troein M, Rastran L, Selander S. Health beliefs and heart disease risk among middle-aged Swedish men. Results from screening in an urban primary care district. Scand J Prim Health Care 1997;15:198-202.

26. Swift SC, Armstrong JE, Beerman KA, Campbell RK, Pond-Smith D. Attitudes and beliefs about exercise among persons with non-insulin dependent diabetes. Diabetes Educ 1995;21:533-40.

27. Becker $\mathrm{MH}$, ed. The health belief model and personal health behavior. New York: Charles B. Slack; 1974.

28. Rosenstock IM, Strecher VJ, Becker MH. Social 
learning theory and the Health Belief Model. Health Educ Q 1988;15:175-83.

29. Clark NM, Dodge JA. Exploring self-efficacy as a predictor of disease management. Health Educ Behav 1999;26:78-89.

30. McDonald-Miszczak L, Maki SA, Gould ON. Selfreported medication adherence and health status in late adulthood: the role of beliefs. Exp Aging Res 2000;26:189-207.

31. Love MM, Pearce KA, Williamson MA, Barron MA, Shelton BJ. Patients, practices and relationships: challenges and lessons learned from the KAN CaRESS clinical trial. J Am Board Fam Med 2006;19: 75-84.

32. Doherty WJ, Schrott HG, Metclaf L, Iasiello-Vailas L. Effect of spouse support and health beliefs on medication adherence. J Fam Pract 1983;17:837-41.

33. Antonucci TC, Johnson EH. Conceptualization and methods in social support theory and research as related to cardiovascular disease. In: Shumaker SA, Czajkowski SM (eds.). Social support and cardiovascular disease. New York: Plenum; 1994:21-39.

34. Haynes RB. A critical review of the "determinants" of patient compliance with therapeutic regimens. In Sackett DL, Haynes RB (eds). Compliance with therapeutic regimens. Baltimore (MD): Johns Hopkins University Press; 1976:26-39.

35. American Diabetes Association. Standards of medical care in diabetes. Diabetes Care 2007;30:S4-S41.

36. American Diabetes Association. Management of dyslipidemia in adults with diabetes. Diabetes Care 2000;23:S57-S60.

37. Morisky DE, Green LW, Levine DM. Concurrent and predictive validity of a self-reported measure of medication adherence. Med Care 1986;24:67-74.

38. Ware JE Jr, Sherbourne CD. The MOS 36-item short-form health survey (SF-36) I. Conceptual framework and item selection. Med Care 1992;30: 473-83.

39. Ware JE Jr, Kosinski M, Dewey JE. How to score version 2 of the SF-36 Health Survey. Lincoln (RI): QualityMetric, Inc.; 2000.

40. Reporting Measures for the CAHPS Health Plan Survey 3.0. From the CAHPS Health Plan Survey and Reporting Kit 2007. Rockville (MD): Agency for Healthcare Research and Quality (AHRQ); 2006. Available at: https://www.cahps.ahrq.gov/cahpskit/ files/cd108_Reporting_Measures_for_theHealthPlanSurvey.htm. Accessed 12 May 2008.

41. Smith MS, Wallston KA, Smith CA. The development and validation of the Perceived Health Competence Scale. Health Educ Res 1995;10:51-64.
42. Donner A, Klar N. Design and analysis of cluster randomization trials in health research. London (UK): Arnold; 2000.

43. Chlebowy DO, Garvin BJ. Social support, self-efficacy, and outcome expectations: impact on self-care behaviors and glycemic control in Caucasian and African American adults with type 2 diabetes. Diabetes Educ 2006;32:777-86.

44. Verheijden M, Bakx JC, Akkermans R, et al. Webbased targeted nutrition counseling and social support for patients at increased cardiovascular risk in general practice: randomized controlled trial. J Med Internet Res 2004;6:e44.

45. Van Dam HA, van der Horst FG, Knoops L, Ryckman RM, Crebolder HFJM, van den Borne BHW. Social support in diabetes: a systematic review of controlled intervention studies. Patient Educ Couns 2005;59:1-12.

46. DeCoster VA, Cummings SM. Helping adults with diabetes: a review of evidence-based interventions. Health Soc Work 2005;30:259-64.

47. Toljamo M, Hentinen M. Adherence to self-care and social support. J Clin Nurs 2001;10:618-27.

48. Barrera M Jr, Toobert DJ, Angell KL, Glasgow RE, Mackinnon DP. Social support and social-ecological resources as mediators of lifestyle intervention effects for type 2 diabetes. Journal Heath Psychol 2006;11:483-95.

49. Wagner EH, Grothaus LC, Sandhu N, et al. Chronic care clinics for diabetes in primary care. A system-wide randomized trial. Diabetes Care 2001; 25:695-700.

50. Kirsh S, Watts S, Pascuzzi K, et al. Shared medical appointments based on the chronic care model: a quality improvement project to address the challenges of patients with diabetes with high cardiovascular risk. Qual Saf Health Care 2007;16:349-53.

51. Goetzel RZ, Ozminkowski RJ. The health and cost benefits of work site health-promotion programs. Annu Rev Public Health 2008;29:303-23.

52. DeHaven MJ, Hunter IB, Wilder L, Walton JW, Berry J. Health programs in faith-based organizations: are they effective? Am J Public Health 2004; 94:1030-6.

53. Wilhide S, Henderson T. Community Care of North Carolina: a provider-led strategy for delivering cost-effective medical care to Medicaid beneficiaries. Washington DC: American Academy of Family Physicians; 2006. Available at http://www. aafp.org/online/etc/medialib/aafp_org/documents/ policy/state/medicaid/ncfull.Par.0001.File.tmp/ ncfullreport.pdf. Accessed 12 May 2008. 